\title{
Spin dynamics and magnetoelectric coupling mechanism of $\mathrm{Co}_{4} \mathrm{Nb}_{2} \mathrm{O}_{9}$
}

\author{
Guochu Deng, ${ }^{1, *}$ Yiming Cao, ${ }^{2,3}$ Wei Ren, ${ }^{2}$ Shixun Cao, ${ }^{2, *}$ Andrew J. Studer, ${ }^{1}$ Nicolas Gauthier, ${ }^{4}$ Michel Kenzelmann, ${ }^{4}$ \\ Gene Davidson, ${ }^{1}$ Kirrily C. Rule, ${ }^{1}$ Jason S. Gardner, ${ }^{5}$ Paolo Imperia, ${ }^{1}$ Clemens Ulrich, ${ }^{6}$ and Garry J. McIntyre ${ }^{1}$ \\ ${ }^{1}$ Australian Nuclear Science and Technology Organisation, New Illawarra Road, Lucas Heights NSW 2234, Australia \\ ${ }^{2}$ Department of Physics, International Centre of Quantum and Molecular Structures, and Materials Genome Institute, \\ Shanghai University, Shanghai 200444, China \\ ${ }^{3}$ Centre for Magnetic Materials and Devices \& Key Laboratory for Advanced Functional and Low Dimensional Materials \\ of Yunnan Higher Education Institute, Qujing Normal University, Qujing 655011, China \\ ${ }^{4}$ Laboratory for Neutron Scattering and Imaging, Paul Scherrer Institut, CH-5232 Villigen-PSI, Switzerland \\ ${ }^{5}$ Neutron Group, National Synchrotron Radiation Research Centre, Hsinchu 30076, Taiwan \\ ${ }^{6}$ School of Physics, The University of New South Wales, Kensington NSW 2052, Australia
}

(Received 11 May 2017; published 28 February 2018)

\begin{abstract}
Neutron powder diffraction experiments reveal that $\mathrm{Co}_{4} \mathrm{Nb}_{2} \mathrm{O}_{9}$ forms a noncollinear in-plane magnetic structure with $\mathrm{Co}^{2+}$ moments lying in the $a b$ plane. The spin-wave excitations of this magnet were measured by using inelastic neutron scattering and soundly simulated by a dynamic model involving nearest- and next-nearestneighbor exchange interactions, in-plane anisotropy, and the Dzyaloshinskii-Moriya interaction. The in-plane magnetic structure of $\mathrm{Co}_{4} \mathrm{Nb}_{2} \mathrm{O}_{9}$ is attributed to the large in-plane anisotropy, while the noncollinearity of the spin configuration is attributed to the Dzyaloshinskii-Moriya interaction. The high magnetoelectric coupling effect of $\mathrm{Co}_{4} \mathrm{Nb}_{2} \mathrm{O}_{9}$ in fields can be explained by its special in-plane magnetic structure.
\end{abstract}

DOI: 10.1103/PhysRevB.97.085154

\section{INTRODUCTION}

Multiferroic materials have been investigated extensively over the past two decades due to their potential applications in future electronics, such as storage devices, sensors, etc. [1]. In spite of great progress in application-oriented research in this field [2,3], fundamental studies on the mechanism of magnetoelectric (ME) coupling and the origin of multiferroicity of various multiferroic systems are full of challenges due to the inherent difficulties of this topic [4-8].

Recently, $\mathrm{Co}_{4} \mathrm{Nb}_{2} \mathrm{O}_{9}$ (CNO) was reported to demonstrate a high ME coupling coefficient $[9,10]$. This compound belongs to the space group $P \overline{3} c 1$. As shown in Fig. 1(a), $\mathrm{Co}^{2+}$ ions split into $\mathrm{Co} 1$ and $\mathrm{Co} 2$ sites and form chains along the $c$ axis with alternating spacings. The octahedra on the Col sites connect into a nearly planar network by edge-sharing while those on the $\mathrm{Co} 2$ sites join into a buckled network by corner-sharing. These two networks stack along the $c$ axis alternatively. $\mathrm{Co}_{4} \mathrm{Nb}_{2} \mathrm{O}_{9}$ undergoes an antiferromagnetic phase transition at a low temperature $T_{N}(\sim 28 \mathrm{~K})$. Surprisingly, the observed ME coupling effect $[9,10]$ and magnetization measurements [11] are contradictive to the magnetic structures previously proposed by Bertaut et al. [12] and recently by Khanh et al. [13]. The former claimed a collinear magnetic structure with $\mathrm{Co}^{2+}$ moments aligning along the $c$ axis [12], while the latter suggested that magnetic moments lie primarily in the $a b$ plane with certain canting angles toward the $c$ axis [13]. We have measured the magnetization of single-crystal samples along

\footnotetext{
*Corresponding authors: guochu.deng@ansto.gov.au; sxcao@shu.edu.cn
}

different crystallographic axes. The results clearly show a cusp in the data measured along the $a$ axis but no abnormality along the $c$ axis near $T_{N}[11,14]$. This suggests that the magnetic moments do not have components along the $c$ axis, which is incompatible with the two magnetic structure models mentioned above. Thus, the previous interpretations to the ME coupling mechanism in $\mathrm{CNO}$ should be reconsidered too due to the lack of accurate magnetic structure for this compound.

In this paper, we discover an in-plane noncollinear magnetic structure for $\mathrm{CNO}$ by using neutron powder diffraction and irreducible representation analysis. We also propose a dynamic model, which simulates the inelastic neutron scattering data. It indicates that large in-plane anisotropy primarily causes the easy-plane magnetic structure, the Goldstone mode, and the gapped mode in the spin-wave spectrum. Similarly, the Dzyaloshinskii-Moriya (DM) interaction is crucial to the noncollinearity of the spin configuration. Bearing this in-plane noncollinear magnetic structure in mind, we discuss the origin of the ME coupling of CNO in magnetic fields in depth.

\section{EXPERIMENT}

$\mathrm{Co}_{4} \mathrm{Nb}_{2} \mathrm{O}_{9}$ powder and single-crystal samples were prepared by a solid-state reaction and the optical floating-zone method, respectively, at Shanghai University [11]. The neutron powder diffraction experiment was carried out on the high-flux neutron diffractometer WOMBAT [15] at the OPAL reactor of the Australian Nuclear Science and Technology Organisation (ANSTO) using a nominal wavelength of $\lambda=2.41 \AA$ in the temperature range from 5 to $50 \mathrm{~K}$. The irreducible representation analysis was performed using SARAh [16]. 


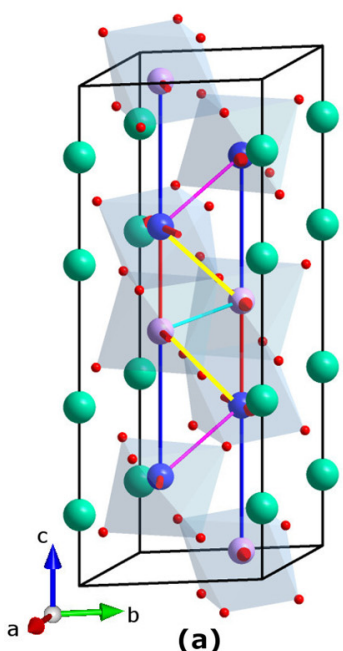

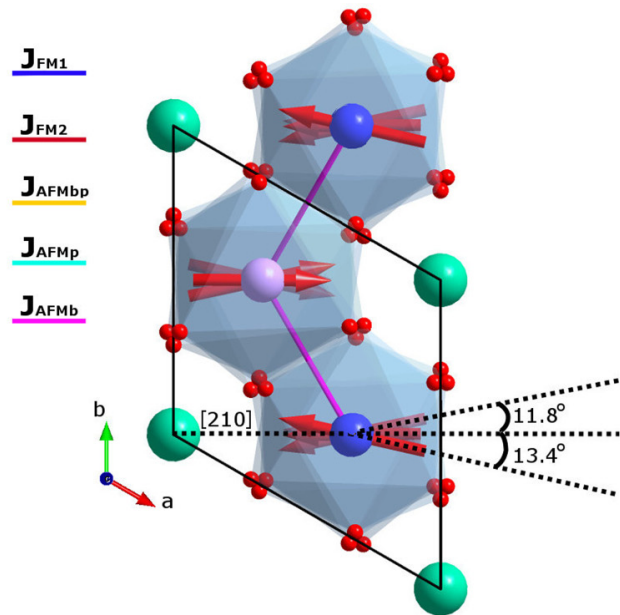

(b)

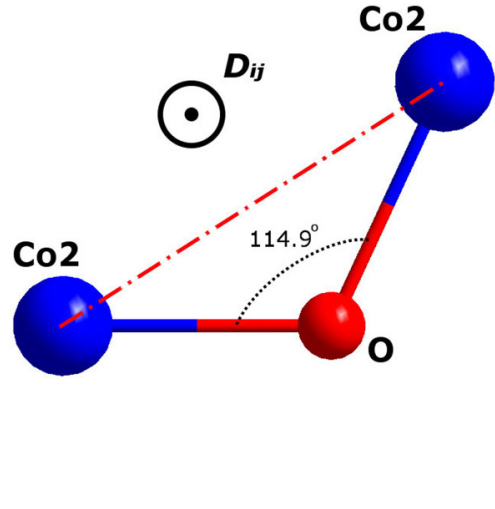

(c)

FIG. 1. (a) Crystal and magnetic structure models of CNO; (b) the canting of magnetic moments in the $a b$ plane; (c) the bond angle of $\mathrm{Co} 2-\mathrm{O} 1-\mathrm{Co} 2$, which is the origin of the DM interaction. In these figures, red spheres denote oxygen atoms, green spheres denote niobium atoms, and purple and blue represent $\mathrm{Co} 1$ and $\mathrm{Co} 2$ atoms, respectively. The red arrows show the orientation of magnetic moments, which are in the $a b$ plane with certain canting angles. The color lines between $\mathrm{Co}^{2+}$ show the exchange interactions. The values in (b) are the angles between the magnetic moments of $\mathrm{Co} 2$ and the [210] direction.

The crystal and magnetic structure models were refined by the Rietveld method using the program FULLPROF [17,18].

Inelastic neutron scattering experiments were performed on both the thermal-neutron and cold triple-axis spectrometers, TAIPAN [19] and SIKA [20] at OPAL, respectively. On TAIPAN, the instrument was configured with an open-openopen-open collimation and a double focus monochromator and analyzer using the fixed $E_{f}=14.87 \mathrm{meV}$. On SIKA, $60^{\prime}-60^{\prime}-60^{\prime}-60^{\prime}$ collimation was used with $E_{f}=8.07 \mathrm{meV}$. A single-crystal sample of CNO with a mass of about $2 \mathrm{~g}$ was mounted on an Al sample holder and aligned in the $a c$ plane for the experiments. Most of the data were collected near the antiferromagnetic zone center (100) by constant- $Q$ scans. The spin-wave dispersion was simulated using the spin-wave calculation package SPINW [21].

\section{MAGNETIC ORDER}

Subtracting the neutron powder diffraction pattern measured above $T_{N}$ from that below $T_{N}$ clearly shows magnetic Bragg peaks in the low- $Q$ range. All magnetic peaks overlap with the nuclear Bragg peaks, indicating that the propagation vector of the ordered magnetic phase is $\boldsymbol{k}=(0,0,0)$. The magnetic $\mathrm{Co}^{2+}$ ions occupy two different sites, namely $(1 / 3,2 / 3,0.017)$ and $(2 / 3,1 / 3,0.3078)$, in CNO. The detailed crystal structure is given in Table II of the Supplemental Material [22].

According to the analysis, the magnetic representation of CNO can be decomposed into six irreducible representations (in short, Irreps), $\Gamma=\Gamma_{1}+\Gamma_{2}+\Gamma_{3}+\Gamma_{4}+2 \Gamma_{5}+2 \Gamma_{6}$, as shown in Table I of the Supplemental Material [22]. Among them, the basis vectors of the first four Irreps $\left(\Gamma_{1}-\Gamma_{4}\right)$ are parallel or antiparallel to the hexagonal axis since these Irreps still keep the threefold rotation symmetry. These four Irreps denote, respectively, four spin configurations $[(++--)$, $(+--+),(++++)$, and $(+-+-)]$ on one chain.
The other two Irreps $\left(\Gamma_{5}\right.$ and $\left.\Gamma_{6}\right)$ have the basis vectors in the $a b$ plane with breaking of the threefold rotation symmetry. In other words, magnetic moments of these two Irreps can lie only in the $a b$ plane.

Considering all these Irreps suggested by SARAh [16], we found that $\Gamma_{6}$ gives the best fit to the observed diffraction pattern (Fig. 2). This refinement generates a noncollinear magnetic structure as shown in Figs. 1(a) and 1(b). The agreement indices of the refinement are $R_{\mathrm{p}}=3.23, R_{\mathrm{wp}}=4.11$, magnetic $R_{\mathrm{B}}=7.08$, and $\chi^{2}=2.92$. The magnetic moments on both the $\mathrm{Co} 1$ and $\mathrm{Co} 2$ sites lie in the $a b$ plane. However, the magnetic moments within each set of sites ( $\mathrm{Co} 1$ and $\mathrm{Co} 2)$

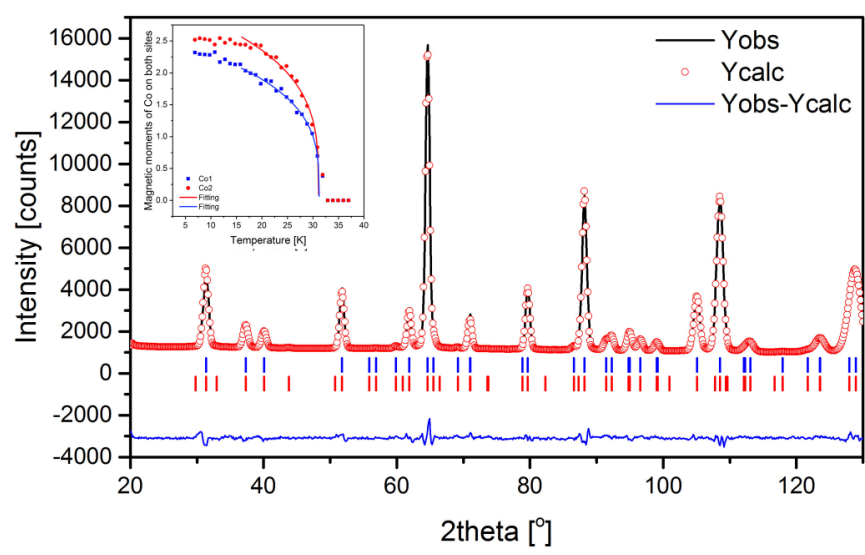

FIG. 2. Neutron powder diffraction pattern at $5 \mathrm{~K}$ from the experiment (black curve) and the Rietveld refinement (red circles) with the Irreps $\Gamma_{6}$; the red tick marks below the data show the nuclear Bragg peak positions of CNO, while the blue tick marks show the magnetic Bragg peak positions of the antiferromagnetic phase. Inset: the temperature dependencies of the $\mathrm{Co} 1$ and $\mathrm{Co} 2$ magnetic moments obtained by fitting the diffraction data. The critical exponents are 0.29(2) and 0.28(2) for the Co1 and Co2 sites, respectively. 

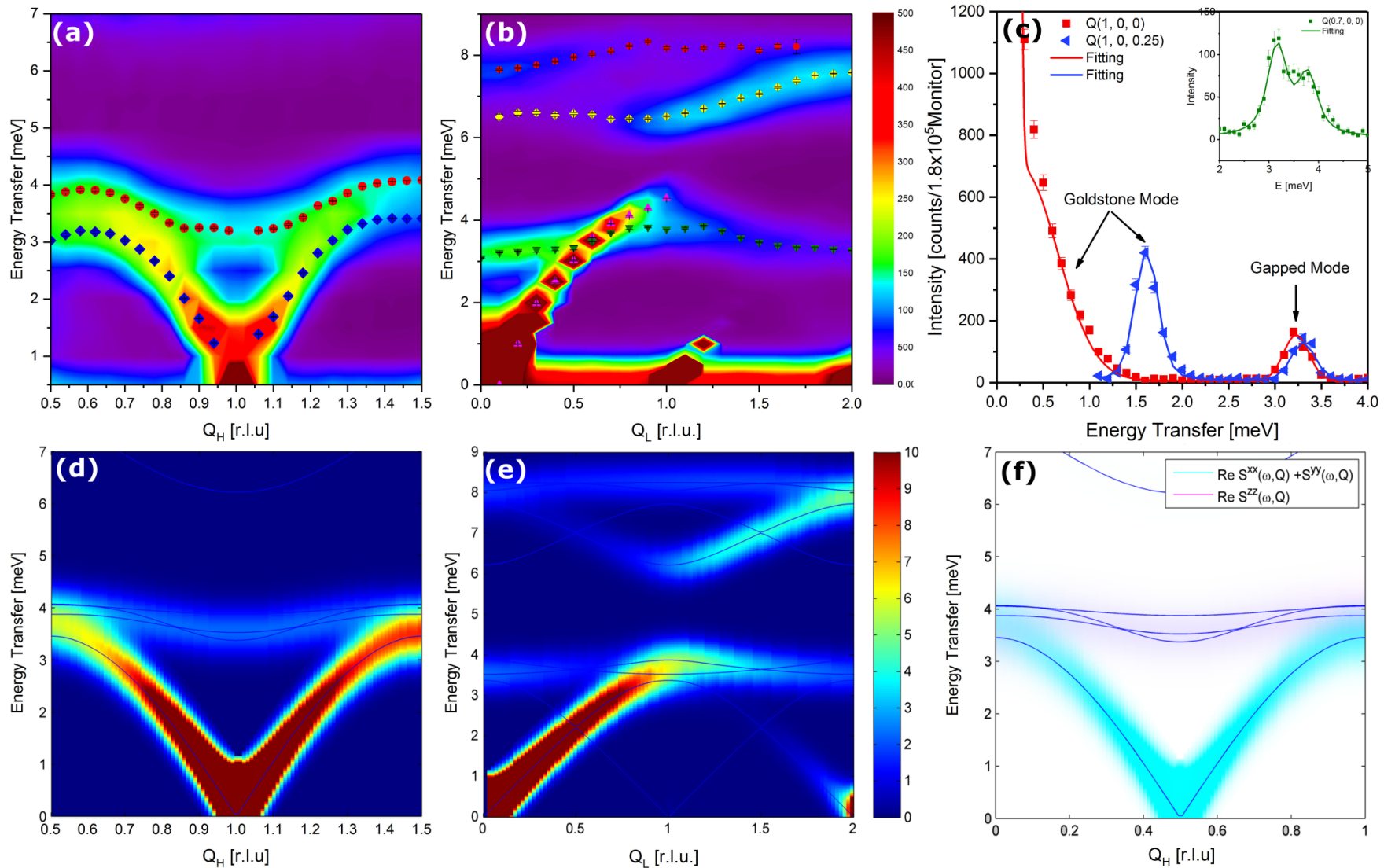

FIG. 3. Spin-wave dispersion measured near the zone center (100) along the (a) $Q_{H}$ and (b) $Q_{L}$ directions in CNO single crystal (from TAIPAN). The dots are the fitted peak positions by convoluting with the instrument resolution. (c) The energy scans at different longitudinal or transverse $Q$ positions (from SIKA). The solid lines correspond to the fittings where the convolution with the instrument resolution was taken into account. Parts (d) and (e) shows the simulated spin-wave spectra along the $Q_{H}$ and $Q_{L}$ directions, respectively, using the dynamic model described in the text. (f) The simulated in-plane and out-of-plane components of the excitation.

are not collinear in the plane, but slightly cant from each other. The average direction of each set of moments is roughly along the [210] direction. The canting angle of the neighbor Co1 moments is $1.3^{\circ} \pm 0.1^{\circ}$ while the canting angle of the Co2 moments is much larger, $25.2(1)^{\circ}\left[=11.8^{\circ}+13.4^{\circ}\right.$; see Fig. 1(b)]. The refined magnetic moments on the Co1 and Co2 sites are $2.32(1) \mu_{B}$ and $2.52(1) \mu_{B}$, respectively.

Our magnetic structure model is different from the two previously reported magnetic structures [12,13], although it is slightly similar to the one proposed by Khanh et al. [13]. It is worthwhile to compare Khanh et al.'s model and ours in detail. First of all, the main magnetic components of both models are not along the $c$ axis. According to our results, the magnetic moments are purely in the $a b$ plane while Khanh et al.'s are nearly in the $a b$ plane with a canting toward the $c$ axis. Secondly, the in-plane magnetic components of both models are roughly aligned along the [210] direction. In our model, however, the magnetic moments cant in the $a b$ plane. In contrast, the moments in Khanh et al.'s model are collinear in the $a b$ plane [13]. Thirdly, the moment magnitudes are similar on both the Co1 and $\mathrm{Co} 2$ sites in our model, while the magnetic moment on the $\mathrm{Co} 2$ sites $\left(3.5 \mu_{B}\right)$ is far larger than the one on the Co1 sites $\left(2.6 \mu_{B}\right)$ in Khanh et al.'s model. Finally, the magnetic moments canting toward the $c$ axis in Khanh et al.'s model [13] are completely contradictive to our magnetization measurements and incompatible with the irreducible representation analysis (see Table I in the Supplemental Material [22]). A recent theoretical calculation by Solovyev et al. [23] indicates that the canting angle should be no more than $2^{\circ}$ if canting exists, which is substantially smaller than the canting angles $22^{\circ}$ and $24^{\circ}$ in Khanh et al.'s model. This theoretical work hints that the in-plane magnetic structure is more favorable with regard to energy. Refining our data using Khanh et al.'s model gave $R_{\mathrm{p}}=3.56, R_{\mathrm{wp}}=4.86$, magnetic $R_{\mathrm{B}}=11.52$, and $\chi^{2}=4.07$, which are considerably larger than the corresponding values obtained with our model. Taking all the arguments above into account, we conclude that the magnetic structure of $\mathrm{CNO}$ should have no canting moment along the $c$ axis.

\section{SPIN DYNAMICS}

The results from the inelastic neutron scattering experiment of CNO are presented in Figs. 3(a) and 3(b). In Fig. 3(a), the dispersion curves of the spin-wave excitation of $\mathrm{CNO}$ were measured along the $Q_{H}$ direction at $\sim 5 \mathrm{~K}$. The experimental data show two branches along both the $Q_{H}$ and $Q_{L}$ directions after convolution fitting with the instrumental resolution. The lower branch corresponds to a Goldstone mode, which goes to zero at the zone center and about $3.5 \mathrm{meV}$ at the zone boundary. 
The upper branch is a gapped mode, which has an energy gap of about $3.1 \mathrm{meV}$ at the zone center. Along the $Q_{L}$ direction, both the gapped mode and the Goldstone mode are visible along the $Q_{L}$ direction, as shown in Fig. 3(b). The Goldstone mode extends to slightly higher energy $(\sim 4.3 \mathrm{meV})$ at the zone boundary along the $Q_{L}$ direction. The intensity of this mode drops substantially in the range $Q_{L}>1.0$ r.l.u. while the gapped mode still propagates in the same range with gradually decreasing intensity. Additionally, some optical modes are observed at a higher-energy range from 6 to $8 \mathrm{meV}$ along the $Q_{L}$ direction. Figure 3(c) shows the energy scans at three different $Q$ positions with higher resolution on SIKA, where both the Goldstone and the gapped modes can be clearly seen.

The rather large energy gap $(>3 \mathrm{meV})$ indicates a contribution not from a DM interaction but from single-ion anisotropy since the DM interaction is usually much weaker. The coexistence of the Goldstone and gapped modes in CNO excludes the possibility that $\mathrm{CNO}$ is an easy-axis magnet like $\mathrm{MnF}_{2}$ [24]. The fact that the magnetic moments of CNO lie in the $a b$ plane indicates it is an easy-plane magnet. Clearly, the Goldstone mode corresponds to an in-plane spin excitation [25], and the gapped mode is related to an out-of-plane spin excitation.

To build a dynamic model to understand the dispersion data in Figs. 3(a) and 3(b), we carefully consider the interactions between nearest and next-nearest neighbors ( $\mathrm{NN}$ and $\mathrm{NNN}$, respectively) of the $\mathrm{Co}^{2+}$ ions in CNO. First, our diffraction data indicate that the distances between $\mathrm{NN}$ and NNN Co1 and Co2 ions are 2.92 and $4.15 \AA$ along the $c$ axis, respectively. The superexchange pathways for the $\mathrm{NN}$ and $\mathrm{NNN}$ interactions $\left(J_{\mathrm{FM} 1}\right.$ and $J_{\mathrm{FM} 2}$ ) run through the bonds Co1-O-Co2 and Co1-OO-Co2, respectively (see Fig. S1 in the Supplemental Material [22]). All bond angles on both of these pathways are close to $90^{\circ}$ (see Table II in the Supplemental Material [22]). According to the Goodenough-Kanamori-Anderson (GKA) rule, both $J_{\mathrm{FM} 1}$ and $J_{\mathrm{FM} 2}$ should therefore be ferromagnetic [26]. This is consistent with our magnetic structure, in which magnetic moments on the same chain align roughly in parallel.

Secondly, we consider the exchange pathways on the planar buckled networks, and in between. The interaction $\left(J_{\mathrm{FMp}}\right)$ between any two neighbor Co1 ions in the planar network takes place through a similar $\sim 90^{\circ} \mathrm{Co} 1-\mathrm{O}-\mathrm{Co} 1$ superexchange pathway, which should be ferromagnetic according to the GKA rule [26]. However, the magnetic structure shows that the magnetic moments on these two sites are roughly antiparallel. Thus, we speculate that this interaction might be very weak. The NN interaction $\left(J_{\mathrm{AFMb}}\right)$ on the buckled network goes through a Co2-O-Co2 pathway with a bond angle of $\sim 115^{\circ}$ [see Fig. 1(c)], resulting in an antiferromagnetic interaction according to the GKA rule [26]. The exchange $J_{\mathrm{AFMbp}}$ between $\mathrm{Co} 1$ and $\mathrm{Co} 2$ takes place through a Co1-O-Co2 bond with an angle of $\sim 129.5^{\circ}$, which should be antiferromagnetic. The colored lines in Figs. 1(a) and 1(b) show all the exchange pathways discussed above [22].

Considering the single-ion anisotropy and DM interaction as well, the Hamiltonian for this Heisenberg magnet reads

$$
\begin{aligned}
H= & J_{\mathrm{FM} 1} \sum_{\langle i, j\rangle} \overrightarrow{\hat{\boldsymbol{S}}}_{i}^{\mathrm{I}} \cdot \overrightarrow{\hat{\boldsymbol{S}}}_{j}^{\mathrm{II}}+J_{\mathrm{FM} 2} \sum_{\langle i, j\rangle} \overrightarrow{\hat{\boldsymbol{S}}}_{i}^{\mathrm{I}} \cdot \overrightarrow{\hat{\boldsymbol{S}}}_{j}^{\mathrm{II}}+J_{\mathrm{FMp}} \sum_{\langle i, j\rangle} \overrightarrow{\hat{\boldsymbol{S}}}_{i}^{\mathrm{I}} \cdot \overrightarrow{\hat{\boldsymbol{S}}}_{j}^{\mathrm{I}}+J_{\mathrm{AFMb}} \sum_{\langle i, j\rangle} \overrightarrow{\hat{\boldsymbol{S}}}_{i}^{\mathrm{II}} \cdot \overrightarrow{\hat{\boldsymbol{S}}}_{j}^{\mathrm{II}}+J_{\mathrm{AFMbp}} \sum_{\langle i, j\rangle} \overrightarrow{\hat{\boldsymbol{S}}}_{i}^{\mathrm{I}} \cdot \overrightarrow{\hat{\boldsymbol{S}}}_{j}^{\mathrm{II}} \\
& +\sum_{\mathrm{Co} 1} D_{1}\left(S_{i Z}^{\mathrm{I}}\right)^{2}+\sum_{\mathrm{Co} 2} D_{2}\left(S_{i Z}^{\mathrm{II}}\right)^{2}+\sum_{\langle i, j\rangle} D_{i j} \cdot\left(\overrightarrow{\hat{\boldsymbol{S}}}_{i}^{\mathrm{II}} \times \overrightarrow{\boldsymbol{S}}_{j}^{\mathrm{II}}\right),
\end{aligned}
$$

where $J_{\mathrm{FM} 1}$ and $J_{\mathrm{FM} 2}$ are the ferromagnetic exchange interactions of $\mathrm{NN}$ and $\mathrm{NNN}$ along $c ; J_{\mathrm{FMp}}, J_{\mathrm{AFMb}}$, and $J_{\mathrm{AFMbp}}$ are the antiferromagnetic exchange interactions of the "planar" networks, the "buckled" networks, and between these two networks, respectively; I and II denote the Co1 and Co2 sites, respectively; $D_{1}$ and $D_{2}$ are positive, indicating easy-plane anisotropies of the Co1 and Co2 sites, respectively; and $D_{i j}$ is the DM interaction.

Since Co1 and $\mathrm{Co} 2$ moments are quite similar in size and orientation, we assume that $D_{1}$ and $D_{2}$ take the same value. The DM interaction is temporarily neglected because it is normally much weaker than the exchange parameters and anisotropy. With this simplified model, we simulated the spin-wave dispersion by using SPINW [21]. The optimized simulation results along the $Q_{H}$ and $Q_{L}$ directions are shown in Figs. 3(d) and 3(e), respectively. The parameters used for this simulation are $J_{\mathrm{FM} 1}=-0.70 \mathrm{meV}$, $J_{\mathrm{FM} 2}=-0.15 \mathrm{meV}, J_{\mathrm{FMp}}=-0.16 \mathrm{meV}, J_{\mathrm{AFMb}}=0.42 \mathrm{meV}$, $J_{\mathrm{AFMbp}}=0.52 \mathrm{meV}$, and $D_{1}=D_{2}=1.8 \mathrm{meV}$. These results indicate that the single-ion anisotropy is quite large in CNO, much larger than other exchange interactions. It is not unusual to observe large spin anisotropy in cobaltites. For exam- ple, $\mathrm{Ba}_{2} \mathrm{CoGe}_{2} \mathrm{O}_{7}$ undergoes an easy-plane antiferromagnetic phase transition at $\sim 7 \mathrm{~K}$ [27]. According to the inelastic neutron scattering experiment and theoretical simulation [27,28], the $\mathrm{NN}$ interaction $J_{\mathrm{AFM}}$ in $\mathrm{Ba}_{2} \mathrm{CoGe}_{2} \mathrm{O}_{7}$ is about $0.2 \mathrm{meV}$ while the single-ion anisotropy $D$ is about $1.15 \mathrm{meV}$. The large anisotropy value is comparable to that of $\mathrm{CNO}$ in this study.

Comparing with Figs. 3(a) and 3(b), the simulations in Figs. 3(d) and 3(e) reproduce almost all the features of the experimental results, for example the Goldstone and gapped modes. In Fig. 3(f), the Goldstone mode corresponds to the in-plane spin excitation while the gapped mode originates from the out-of-plane spin excitation [22]. The intensities of the simulated dispersion curves also agree well with the experiment over the whole $Q$ range, especially reproducing the low intensity in the range $Q_{L}>1.0$ r.l.u. This consistency suggests that our model describes this magnetic system very well.

In this model, a collinear magnetic ground state is established by using the simulated annealing method in SPINW. This disagrees with our magnetic structure model from powder diffraction. If we introduce a small DM interaction into this model through the Co2-O-Co2 path [see Fig. 1(c) and the 
further discussion in the next section], the simulated annealing produces a magnetic structure with different canting angles for moments on both the $\mathrm{Co} 1$ and $\mathrm{Co} 2$ sites [21]. The canting angles are dependent on the value of the DM interaction. A value of $\mathrm{DM} \approx 0.22 \mathrm{meV}$ is able to generate similar canting angles for the $\mathrm{Co} 1$ and $\mathrm{Co} 2$ sites (roughly $\sim 5^{\circ}$ and $\sim 23^{\circ}$, respectively), close to our magnetic structure model. The comparison between our magnetic structure model and the simulated ground-state magnetic structure is shown in Fig. S2 in the Supplemental Material [22]. The similarity between them strongly indicates that the DM interaction is indispensable to understand the noncollinearity of the magnetic structure of CNO. It is worthwhile to point out that introducing the DM interaction as above does not visibly change the simulated spin-wave dispersion in Figs. 3(d)-3(f).

\section{ORIGIN OF THE MAGNETOELECTRIC EFFECT}

In our magnetic structure model, $\boldsymbol{S}_{i}$ and $\boldsymbol{S}_{j}$ are in the $a b$ plane and are noncollinear. Thus, their product should be a vector along the $c$ axis. Since $\boldsymbol{e}_{i j}$ is a unit vector from $\boldsymbol{S}_{i}$ to $\boldsymbol{S}_{j}$, which is in the (110) plane (or any equivalent planes), the polarization $\boldsymbol{P}$ should be along the [110] (or any equivalent) direction because $\boldsymbol{P}$ is proportional to $\boldsymbol{e}_{i j} \times\left(\boldsymbol{S}_{i} \times \boldsymbol{S}_{j}\right)$ according to the spin-current model [7]. However, $\boldsymbol{P}$ is canceled by the neighbor polarization $\boldsymbol{P}^{\prime}$ due to the antiparallel spin configuration. This agrees with the zero polarization at zero field reported in the literature $[10,13]$.

What happens when applying a magnetic field? According to Chubokov [29], the magnetic moments of a hexagonal in-plane magnet stay in-plane even when applying a strong out-of-plane magnetic field if its in-plane anisotropy $D$ is much larger than the dominant exchange interaction. Therefore, we only consider the in-plane field effect here. In an in-plane magnetic field, magnetic moments will rotate slightly to reduce their angles to the external field direction, which causes nonequivalent $\boldsymbol{P}$ and $\boldsymbol{P}^{\prime}$. Consequently, the total polarization will be nonzero. In a higher field, both $\boldsymbol{P}$ and $\boldsymbol{P}^{\prime}$ point to the same direction due to the spin-flip transition, resulting in an enhanced polarization. More details on the field effect are given in the Supplemental Material (see Fig. S3) [22]. According to this understanding, interestingly, regardless of which direction the external field is along, the total polarization always remains along the [110] or any equivalent directions. This is determined by the magnetic structure of CNO itself. Our analysis is strongly supported by the fact that the maximal polarization is always observed along the [110] direction, as reported by Khanh et al. [13].

The spin-current model is also called the inverse-DM effect. It is interesting to know which bond causes this effect in CNO. The pathway Co2-O-Co2 forms a triangle with a bond angle of $\sim 115^{\circ}$. $\mathrm{Co} 2$ moments have larger canting angles than Co1 moments on the Co1-O-Co1 bond. Therefore, we conclude that the DM interaction takes place through the $\mathrm{Co} 2-\mathrm{O}-\mathrm{Co} 2$ bond rather than through the Co1-O-Co1 bond. The dynamic model involving DM on the Co2-O-Co2 bond generates a noncollinear magnetic ground state, in agreement with our magnetic structure model determined from powder diffraction (see Fig. S2 in the Supplemental Material [22]). The spin canting angles on the Co1 sites are smaller than those on the $\mathrm{Co} 2$ sites. On the contrary, a DM interaction on the Co1-O-Co1 bond produces an opposite result. Therefore, it is the $\mathrm{Co} 2-\mathrm{O}-\mathrm{Co} 2$ bond that contributes to the DM interaction in $\mathrm{CNO}$, which finally causes the strong ME coupling.

\section{CONCLUSION}

The magnetic structure and spin dynamics of $\mathrm{CNO}$ were studied by neutron powder diffraction and inelastic neutron scattering, respectively. The dynamic behaviors, such as the existence of the Goldstone and the gapped modes, reveal that CNO is an easy-plane magnet with large easy-plane anisotropy. The DM interaction causes the noncollinear in-plane magnetic structure of CNO. The high ME coupling effect of CNO can be explained entirely by the spin-current model. Our proposed mechanism can be widely used to explain the ME coupling effect in other easy-plane magnets. Therefore, this study also provides a guideline to search for novel ME material candidates in hexagonal easy-plane magnets.

\section{ACKNOWLEDGMENTS}

We thank ANSTO for the allocation of neutron beam time on WOMBAT, SIKA, and TAIPAN (P3843, P3844, P4348, and P4851). This work is supported by the National Natural Science Foundation of China (NSFC, No. 11774217, No. 51372149, and No. 51672171), the National Key Basic Research Program of China (Grant No. 2015CB921600), Eastern Scholar Program from Shanghai Municipal Education Commission, and the research grant (No. 16DZ2260600) from Science and Technology Commission of Shanghai Municipality.
[1] J. F. Scott, Nat. Mater. 6, 256 (2007).

[2] R. Ramesh and N. A. Spaldin, Nat. Mater. 6, 21 (2007).

[3] S.-W. Cheong and M. Mostovoy, Nat. Mater. 6, 13 (2006).

[4] J. H. Lee, Y. K. Jeong, J. H. Park, M. A. Oak, H. M. Jang, J. Y. Son, and J. F. Scott, Phys. Rev. Lett. 107, 117201 (2011).

[5] R. D. Johnson, N. Terada, and P. G. Radaelli, Phys. Rev. Lett. 108, 219701 (2012).

[6] M. Kenzelmann, A. B. Harris, S. Jonas, C. Broholm, J. Schefer, S. B. Kim, C. L. Zhang, S.-W. Cheong, O. P. Vajk, and J. W. Lynn, Phys. Rev. Lett. 95, 087206 (2005).
[7] H. Katsura, N. Nagaosa, and A. V. Balatsky, Phys. Rev. Lett. 95, 057205 (2005).

[8] I. A. Sergienko and E. Dagotto, Phys. Rev. B 73, 094434 (2006).

[9] T. Kolodiazhnyi, H. Sakurai, and N. Vittayakorn, Appl. Phys. Lett. 99, 132906 (2011).

[10] Y. Fang, Y. Q. Song, W. P. Zhou, R. Zhao, R. J. Tang, H. Yang, L. Y. Lv, S. G. Yang, D. H. Wang, and Y. W. Du, Sci. Rep. 4, 3860 (2014).

[11] Y. Cao, Y. Yang, M. Xiang, Z. Feng, B. Kang, J. Zhang, W. Ren, and S. Cao, J. Cryst. Growth 420, 90 (2015). 
[12] E. F. Bertaut, L. Corliss, F. Forrat, R. Aleonard, and R. Pauthenet, J. Phys. Chem. Solids 21, 234 (1961).

[13] N. D. Khanh, N. Abe, H. Sagayama, A. Nakao, T. Hanashima, R. Kiyanagi, Y. Tokunaga, and T. Arima, Phys. Rev. B 93, 075117 (2016).

[14] Y. Cao, G. Deng, P. Beran, Z. Feng, B. Kang, J. Zhang, N. Guiblin, B. Dkhil, W. Ren, and S. Cao, Sci. Rep. 7, 14079 (2017).

[15] A. J. Studer, M. E. Hagen, and T. J. Noakes, Physica B 385-386, 1013 (2006).

[16] A. S. Wills, Physica B 276-278, 680 (2000).

[17] H. M. Rietveld, J. Appl. Cryst. 2, 65 (1969).

[18] J. Rodriguez-Carvajal, Physica B 192, 55 (1993).

[19] S. A. Danilkin and M. Yethiraj, Neutron News 20, 37 (2009).

[20] C.-M. Wu, G. Deng, J. S. Gardner, P. Vorderwisch, W.-H. Li, S. Yano, J.-C. Peng, and E. Imamovic, J. Instrum. 11, P10009 (2016).

[21] S. Toth and B. Lake, J. Phys.: Condens. Matter 27, 166002 (2015).
[22] See Supplemental Material at http://link.aps.org/supplemental/ 10.1103/PhysRevB.97.085154 for the irreducible representation analysis, the separation of the Goldstone mode and the gapped mode, the superexchange pathway, and a comparison between our magnetic structure model and the calculated magnetic ground state.

[23] I. V. Solovyev and T. V. Kolodiazhnyi, Phys. Rev. B 94, 094427 (2016).

[24] G. G. Low, A. Okazaki, R. W. H. Stevenson, and K. C. Turberfield, J. Appl. Phys. 35, 998 (1964).

[25] A. V. Chubukov, J. Phys.: Condens. Matter. 2, 1593 (1990).

[26] I. de Pedro, J. M. Rojo, J. Rodríguez Fernández, M. T. FernándezDíaz, and T. Rojo, Phys. Rev. B 81, 134431 (2010).

[27] A. Zheludev, T. Sato, T. Masuda, K. Uchinokura, G. Shirane, and B. Roessli, Phys. Rev. B 68, 024428 (2003).

[28] J. Romhanyi and K. Penc, Phys. Rev. B 86, 174428 (2012).

[29] A. V. Chubukov, J. Phys. C 21, L441 (1988). 